\title{
Post-Pandemic Directions for Aging Care Research
}

\author{
Kristine Williams \\ E. Jean Hill Professor \\ University of Kansas School of Nursing
}

7 he COVID-19 pandemic changed the world for everyone, but especially for older adults. With age established as the primary risk factor for severe illness and death, older adults were disproportionately affected (Lebrasseur et al., 2021), and life expectancy declined for the first time in recent history (Andrasfay \& Goldman, 2021). The first reported death from COVID-19 was a nursing home (NH) resident, and to date (September 2021) approximately 25\% of deaths from COVID-19 in the United States were nursing home residents (Abrams, 2021). As the nation emerges from the pandemic, this crisis represents not only the danger of continuing deaths, lingering variants, and pockets of low vaccination rates, but also the impetus and opportunity to improve care for aging adults. Improving ongoing aging care must take into consideration both the lessons learned during the pandemic and the new solutions envisioned. Positive care changes such as telehealth should be continued and further expanded, while new measures to reduce the negative impact of infection and isolation in institutional care settings must be identified and implemented in practice (Convergence, 2020; Edelman et al., 2020).

Healthcare consumers of all ages suffered negative effects of the pandemic due to reduced access and postponement of preventive and health promotion care and elective surgeries. In 2020, cancer screening declined by an estimated $80 \%$, and other preventive and health promotion care such as dental cleaning and community based supportive care programs such as Meals on Wheels and adult day care were halted (Greiner et al., 2021; Lebrasseur et al., 2021). Even exercise was adversely affected as older adults were cautioned to avoid contact with others out of doors, as well as in gyms and group exercise classes. The impact of social isolation may have had the greatest impact on older adults; increased substance use and negative lifestyle changes were common while mental health services became less accessible.

For older adults requiring supportive care in long-term services and support settings (LTSS) ranging from home care

to skilled NH care, dramatic changes occurred (Lebrasseur et al., 2021; U.S. Department of Health and Human Services, 2021). Of the adults 65 and older in the U.S. receiving Medicare, an estimated two out of five recipients who lived in NHs in 2020 were diagnosed with either COVID-19 or likely COVID-19. The number of Medicare $\mathrm{NH}$ beneficiaries dying per day increased by approximately 1,000 in 2020 compared to 2019; overall annual $\mathrm{NH}$ mortality rates increased from $17 \%$ in 2019 to $22 \%$ in 2020 . COVID disproportionately affected $50 \%$ of Black, Hispanic, and Asian NH residents compared to $41 \%$ of white residents (Andrasfay \& Goldman, 2021). Excess deaths and subsequent hesitancy for placing older adults in institutional settings have contributed to estimated declines in occupancy of $25 \%$ for NHs and other LTSS. For those businesses, the decline in occupancy rates is problematic, prompting some facility closures (Lawhorn, 2021). 
However, these dramatic changes may provide impetus for positive changes in provision of care across LTSS.

$\mathrm{NH}$ care has long been identified as lacking quality and person-centeredness. Infection control has been a leading issue with renewed focus during and after the pandemic. The punitive nature of regulations and inspections has been pervasive in NHs, resulting in a culture that does not embrace quality improvement as other healthcare settings have done. NHs have been slow to adopt quality improvement initiatives and have been less likely to change practices based on research evidence. Emerging from the pandemic, $\mathrm{NHs}$ and other settings caring for aging adults have a critical need to invest in and endorse research and the evidence it provides to improve care (Edelman et al., 2020). This paper highlights topic, policy, and infrastructure directions for research to leverage improvement in care for older adults in the post-pandemic world.

\section{Improving Nursing Home Care}

Infection control became an essential priority for $\mathrm{NH}$ care during the COVID-19 pandemic. The Centers for Medicare and Medicaid Services (CMS), which regulates skilled NHs, responded to the early recognition of deaths among $\mathrm{NH}$ residents by mandating personal protective equipment (PPE) use and isolation precautions that undoubtedly prevented even more resident deaths. Initially NHs were challenged with shortages in PPE supplies; currently infection control practice changes are issued frequently, requiring prompt responses by $\mathrm{NH}$ administrators and altered practices for staff.

One sequela of heightened infection control was social isolation experienced by $\mathrm{NH}$ residents whose group activities were suspended. This resulted in lack of socialization for residents and inabil- ity for staff to assist and interact with multiple residents in groups, such as during group dining. Visits from family members that previously supported resident well-being were curtailed, further contributing to resident isolation and increasing dependence on staff for socialization and emotional support. Added time required for putting personal protective equipment on and off when in contact with residents and other infection control precautions limited staff and resident contact, further decreasing interpersonal interactions. Social isolation increases loneliness, contributing to depression, anxiety, fall risk, decline in function, dehydration, malnutrition, behavioral symptoms, and even suicidal ideation (Edelman et al., 2020). Limited mobility from resident confinement to rooms coupled with increased demands on staff time contributed to pressure injury risk, as well as urinary tract infections. Although these physiological and psychosocial issues exacerbated long-standing concerns about $\mathrm{NH}$ quality, they present an opportunity to improve $\mathrm{NH}$ care.

Infection control may be improved by moving to private resident rooms and using technology to connect residents interpersonally and more efficiently to staff or by grouping small numbers of residents in pods, limiting social contacts while optimizing socialization. Increased use of telemedicine within NHs would help overcome the need to access care outside the NH. Increased use of electronic records for charting, not widely implemented in NHs to date, can enable all levels of staff to document and share information electronically, critical for improving communication, clinical decision making, and reporting. Research is needed to explore these opportunities for improvements and how best to implement 
them for positive change. Others suggest that approaches used in other countries and technology common in acute care settings, including extending staff care with monitoring systems and even featuring telepresence interactions with staff, may be adapted to resolve $\mathrm{NH}$ care quality issues (Edelman et al., 2020).

Inadequate staffing was accentuated by the pandemic. Staff who were exposed to or diagnosed with COVID-19 were unable to work. The increased time for attending to PPE and private rooms to provide individual care also increased the workloads for staff. Staff training must be adaptive, focusing on priority issues such as infection control updates. Asynchronous training sessions, such as online education, may best meet the justin-time needs for access by multiple, increasingly busy staff. Training must also focus on making pandemic-level care person-centered, such as in training staff how best to communicate with a resident with dementia or a hearing loss while wearing PPE.

Most direct care staff are certified nursing assistants (CNAs) who have limited training. Over the past year, training requirements were relaxed to make hiring of adequate staff feasible but often overlooking the importance of training. Many CNAs and other NH staff work at multiple care facilities, increasing the risk for spreading infection. Notably, a number of states report that less than $50 \%$ of $\mathrm{NH}$ staff have opted for COVID-19 vaccinations (Data.CMS.org, 2021). These continuing issues have hampered the elimination of COVID from $\mathrm{NH}$ care. Strategies to hire and train direct care staff, limit cross-facility transmission, and promote vaccination need to be addressed by research to identify evidence-based solutions.

NHs serve the frailest older adults, so it is not surprising that NHs have been the most affected of the LTSS. LTSS range from supportive community-based services (home care, Meals on Wheels, adult day care) to congregate senior living settings and assisted living, including memory care. These other settings experienced lower rates of excessive deaths during the pandemic. Approximately one-third of $\mathrm{NH}$ residents died annually prior to COVID. This increased to approximately 60 deaths per 1,000 NH residents. In comparison, community-dwelling older adults died at a rate of six to seven per 1,000, and assisted living deaths reached 19.3 per 1,000 residents during 2020. These statistics suggest the need for research to support older adult residency at home or in less intensive LTSS and for research to develop and test interventions to maintain functional status and ability to manage everyday living, thus reducing progression to higher levels of care (NORC, 2021).

\section{Home}

Improving Care Beyond the Nursing

Rethinking nursing homes in favor of more community-based models has been suggested, and this approach may be timely considering estimates that only $15 \%$ of those 65 and older will not need long-term care as they age. Over $50 \%$ of older adults will require assistance with multiple activities of daily living for over one year (Abrams, 2021; Belbase et al., 2021). Technology advances can help support ongoing community residency; however, the role of technology is limited and cannot totally replace human contact. Policy and current reimbursement barriers must be overcome to increase the availability of care at home. This includes funding to purchase technology to avoid disparities related to the socioeconomic status of many older adults with limited income. Many services deemed 
custodial or not requiring skilled care are not covered or are covered sporadically. For example, extended supportive care is available through Medicaid to prevent $\mathrm{NH}$ placement, but many states limit the number of persons who receive these services, resulting in long waiting lists and unmet needs. Programs such as PACE, the Program of All-Inclusive Care for the Elderly, that integrate medical, behavioral, and social care for frail older adults under a capitated managed care system with a goal of autonomy and maintained community living are showing increasing evidence of success and can serve as a model for potential expansion (Eng et al., 1997). Rural areas often lack the range of services and infrastructure such as broadband access to support telehealth, and these issues also must be addressed.

An estimated 53 million, or one in five, Americans provide care for older adult family members and friends at home, including a large proportion of those diagnosed with dementia (Retirement Research Foundation, 2021). Paying family caregivers is one suggested approach to support home care. Research on how to best support families to reduce LTSS needs is critical (Teshale et al., April 2021). Research and policy must address other innovative approaches to reduce reliance on NH care (Urtamo et al., 2019). Aging research must also focus on interventions to prevent frailty and decline, to help older adults maintain optimal functionality and a level of everyday competence for independent or minimally supported living in quasi-independent, congregate, and assisted living settings (Convergence, 2020).

\section{Changes to Research Infrastructure}

As public health expands in response to the pandemic, a focus on geriatric public health is needed (Wigginton et al., 2020). To meet the needs for quality care for older adults, research scientists and investigators who are focused on this population should be developing and implementing solutions. Funding from the National Institutes of Health is required for research to improve aging care and produce the next generation of gerontologists.

Research processes and the development of new experts must also address the transition from in-person to remote research and overcome restricted access that severely hampered research in NHs during the pandemic. Investigative teams conducting research in LTSS must build on progress in remote conduct of research initiated during the pandemic that also addresses barriers to remote participation in research for older adults (Bertuzzi \& Dirita, 2021; Radecki \& Schonfeld, 2020; Tugend, 2021). Although remote research teams may increase distance collaborations and the number of interdisciplinary teams, research is needed to determine best practices for socializing junior investigators into these teams. NHs and other LTSS also need to endorse and incorporate research and implementation science in these healthcare settings. Including researchers on the clinical team at the point of care has potential to not only efficiently identify needs and design research to improve care, but also facilitate implementation of research evidence by members of the LTSS culture. Cross-training of clinicians in research skills and including those with research expertise on staff may be essential for ongoing improvement in aging care quality across care settings. The crisis of the COVID-19 pandemic provides a wakeup call with new impetus and direction to overcome old and new challenges to provide quality $\mathrm{NH}$ care. 


\section{References}

Abrams, A. (2021). COVID-19 Exposed Faults in America's Elder Care System. This is our best shot to fix them. TIME. https://time.com/6071582/elder-care-after-covid-19/ Andrasfay, T., \& Goldman, N. (2021). Reductions in 2020 US life expectancy due to COVID-19 and the disproportionate impact on the Black and Latino populations. Proceedings of the National Academy of Sciences, 118(5), e2014746118. https://doi.org/10.1073/ pnas. 2014746118

Belbase, A., Chen, A., \& Munnell, A. H. (2021). What Level of Long-Term Services and Supports Do Retirees Need? https://crr.bc.edu/briefs/what-level-of-long-term-services-andsupports-do-retirees-need/

Bertuzzi, S., \& Dirita, V. J. (2021, May 7). After COVID-19 We Will Need a New Research System. We Need to Start Planning Now. mBio. https://journals.asm.org/journal/ $\underline{\text { mbio }}$

Convergence. (2020). Supplement to "Rethinking Care for Older Adults". https://www. johnahartford.org/dissemination-center/view/convergence-report-supplement-to-rethinking-care-for-older-adults

Data.CMS.org. (2021, June 27). COVID-19 Nursing Home Data. Retrieved July 9, 2021 from https://data.cms.gov/stories/s/COVID-19-Nursing-Home-Data/bkwz-xpvg/

Edelman, L. S., McConnell, E. S., Kennerly, S. M., Alderden, J., Horn, S. D., \& Yap, T. L. (2020, May 26). Mitigating the Effects of a Pandemic: Facilitating Improved Nursing Home Care Delivery Through Technology. JMIR Aging, 3(1), e20110. https://doi. org/10.2196/20110

Eng, C., Pedulla, J., Eleazer, G. P., McCann, R., \& Fox, N. (1997). Program of All-inclusive Care for the Elderly (PACE): An Innovative Model of Integrated Geriatric Care and Financing. Journal of the American Geriatrics Society, 45(2), 223-232. https://doi.org/ https://doi.org/10.1111/j.1532-5415.1997.tb04513.x

Greiner, B., Tipton, S., Nelson, B., \& Hartwell, M. (2021, June 21). Cancer screenings during the COVID-19 pandemic: An analysis of public interest trends. Current Problems in Cancer, 100766. https://doi.org/https://doi.org/10.1016/j.currproblcancer.2021.100766 Lawhorn, C. (2021, April 21). Pending Closure of Brandon Woods Nursing Home is expected to create crunch for families seeking care. Lawrence Journal World. https://www2. ljworld.com/

Lebrasseur, A., Fortin-Bédard, N., Lettre, J., Raymond, E., Bussières, E. L., Lapierre, N., Faieta, J., Vincent, C., Duchesne, L., Ouellet, M. C., Gagnon, E., Tourigny, A., Lamontagne, M., \& Routhier, F. (2021, April 12). Impact of the COVID-19 Pandemic on Older Adults: Rapid Review. JMIR Aging, 4(2), e26474. https://doi.org/10.2196/26474

NORC. (2021). The Impact of COVID-19 on Seniors Housing Final Report. https://info.nic. org/hubfs/Outreach/2021 NORC/20210601\%20NIC\%20Final\%20Report\%20and $\% 20$ Executive\%20Summary\%20FINAL.pdf

Radecki, J., \& Schonfeld, R. C. (2020). The Impacts of COVID-19 on the Research Enterprise: A Landscape Review (Research Report, Issue). https://sr.ithaka.org/publications/the-impacts-of-covid-19-on-the-research-enterprise/

Retirement Research Foundation. (2021). Investing in Caregivers: An Essential Resource for our Nation. https://www.rrf.org/introducing-rrfs-second-issue-brief-investing-in-caregivers/ 
Teshale, S., Fox-Grage, W., \& Purington, K. (April 2021). Paying Family Caregivers through Medicaid Consumer-Directed Programs: State Opportunities and Innovations. https:// www.nashp.org/paying-family-caregivers-through-medicaid-consumer-directed-programs-state-opportunities-and-innovations/

Tugend, A. (2021). Lessons From Remote Research. The Chronicle of Higher Education. https://www.chronicle.com/virtual-events/remote2

U.S. Department of Health and Human Services. (2021). (OEI-02-20-00490). (Data Snapshot, Issue). https://oig.hhs.gov/oei/reports/OEI-02-20-00490.asp

Urtamo, A., Jyväkorpi, S. K., \& Strandberg, T. E. (2019). Definitions of successful ageing: a brief review of a multidimensional concept. Acta bio-medica : Atenei Parmensis, 90(2), 359-363. https://doi.org/10.23750/abm.v90i2.8376

Wigginton, N. S., Cunningham, R. M., Katz, R. H., Lidstom, M. E., Moler, K. A., Wirtz, D., \& Zuber, M. T. (2020, June 12). Moving Academic Research Forward during COVID-19. SCIENCE, 368(6496), 1190-1192. https://science.sciencemag.org/ 